\title{
The History of Expansion of the Genus Bursaphelenchus (Nematoda: Aphelenchida: Parasitaphelenchidae)
}

\author{
A. Yu. Ryss $^{a}$, P. Vieira ${ }^{b}$, and M. Mota ${ }^{b}$ \\ Presented by Academician A.F. Alimov September 10, 2007
}

Received September 19, 2007

\section{DOI: $10.1134 / \mathrm{S} 0012496608020087$}

Because of globalization and removal of geographical barriers, frequent biological invasions of introduced species become an urgent environmental problem. According to the Convention on Biological Diversity (CBD), precise identification of dangerous aggressive species at the early stages of their invasion to new regions is the most important component of the environmental control and monitoring. To resist the potential environmental hazard, the precise data are required on the current distribution and history of expansion of pests that are of global economic importance.

We performed biogeographical analysis of phytopathogenic nematodes of the genus Bursaphelenchus, which are of world plant quarantine importance. We analyzed the species groups erected previously on the basis of morphological data and the biotic relationships with the host plants and insect vectors [1]. A model of the genus dispersal based on all data on Bursaphelenchus records in natural biotopes was developed using the secondary Brooks parsimony approach (sBPA).

Two species of the genus are included into the A1 list of especially dangerous plant quarantine organisms [2]. These are Bursaphelenchus xylophilus, the causative agent of the pine wilt disease in Asia and America, and Bursaphelenchus cocophilus, the pathogen of the palm red ring disease in the Caribbean region. The Bursaphelenchus genus includes 78 phytophathogenic species the life cycle of which is associated with woody plants (mostly coniferous trees from the family Pinaceae), insects (beetles from the families Scolytidae, Cerambycidae, Curculionidae, and Buprestidae), and basidium-producing fungi. The insect vectors have played an important role in the Bursaphelenchus evolution: the initial association, Scolytidae

\footnotetext{
${ }^{a}$ Zoological Institute, Russian Academy of Sciences, Universitetskaya nab. 1, St. Petersburg, 199034 Russia

${ }^{b}$ University of Évora, Apartado 94, 7001, Évora, Portugal
}

beetles-Pinaceae conifers, has changed, giving rise to two natural species groups within the genus: the xylophilus group (the vectors were longhorn beetles of the family Cerambycidae) and the hunti group (the vectors belong to the order Hymenoptera) [1].

A modified sBPA was used to determine the specific features of the spread of these nematodes and their relationships with the host plants [3-5]. In works by Brooks and his followers, matrices and dendrograms were obtained by the parsimony method on the basis of well-grounded phylogenies of both plant and animal taxa. The dendrogram branches of the phylogenetic tree of the studied taxon served as fields in matrices for the regions and host taxa. On the basis of these matrices, new dendrograms of the regions and hosts were obtained to develop the concepts of the evolution for the taxa and fauna of the areas under consideration, accurate to species. In this study, the matrices of plants and large regions were developed on the basis of the species groups that are of high prognostic value, rather than on the phylogenetic branches of nematodes (these matrices were based on almost invariable complex traits, namely, the structural details of the male copulative apparatus). Since the detailed phylogenetic model of Bursaphelenchus species is not available so far, the Brooks approach is applicable only in this modification. As a result, the accuracy of historical interpretation is changed: no conclusions on the dispersal history or changes in host specificity of each species can be drawn. However, the major trends in historical development of the genus as a whole and of prognostic species groups can be followed. The dendrogram was constructed using the PAUP ver. 4.0b10 software [6].

Figure 1a shows the similarity dendrogram for the faunae of the genus Bursaphelenchus (at the level of species groups) in world regions. The data on species distribution that were reported earlier in the form of detailed tables by Ryss et al. [1] were generalized to large biogeographical regions for the species groups of the genus (table). Because of heterogeneous populating of North America with Bursaphelenchus species groups (both primitive groups, such as hunti and aber- 

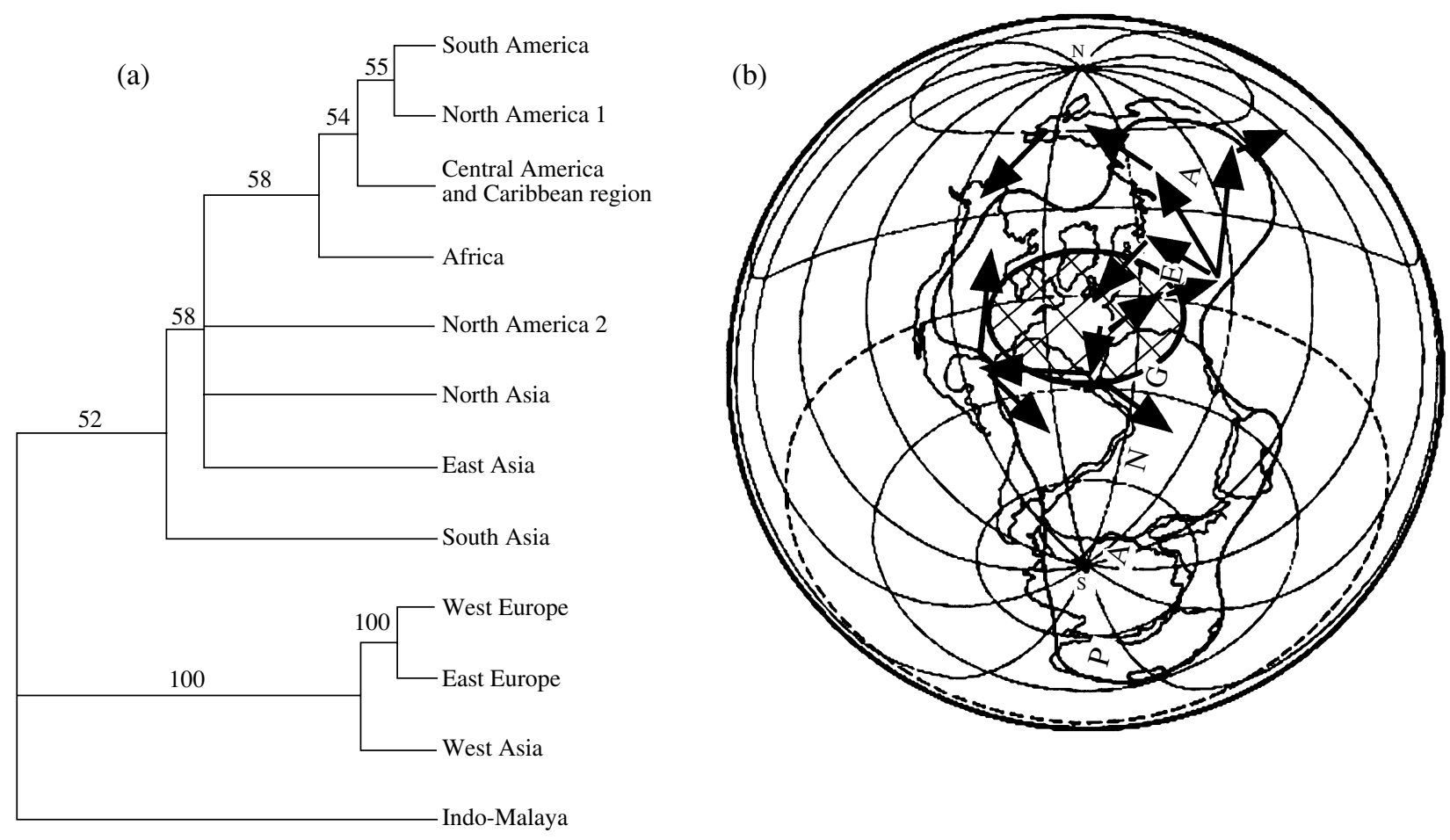

Fig. 1. The history of expansion of species groups of the genus Bursaphelenchus: (a) the similarity dendrogram of the Bursaphelenchus fauna at the level of species groups in the world regions (parsimony, consensus for the best 67 trees, heuristic method, the frequencies of tree nodes are indicated in percent); (b) the history of Bursaphelenchus expansion; the hatched ellipse shows the center of origin of the genus; arrows show the expansion pathways corresponding to the dendrogram branches; the paleogeographical outlines of continents are shown according to [11].

rans, and advanced groups, such as xylophilus and piniperdae, were found), the sBPA technique of dividing heterogeneous groups was used. North America was assumed to contain two biogeographical units, North America 1 and North America 2. The areas lacking
Bursaphelenchus were excluded from the analysis and the table. Heuristic search (parsimony, the branch-andbound algorithm with a maximum of 1000 trees) yielded 67 most economical trees with the following parameters: tree length $=16, C I=0.43, R I=0.64$, and

Distribution of species groups of the genus Bursaphelenchus in the world's regions

\begin{tabular}{l|c|c|c|c|c|c}
\hline \multirow{2}{*}{\multicolumn{1}{c|}{ Region }} & \multicolumn{5}{c}{ Group of species } \\
\cline { 2 - 6 } & hunti G1 & aberrans G2 & eidmanni G3 & borealis G4 & xylophilus G5 & piniperdae G6 \\
\hline Indo-Malaya & 0 & 1 & 0 & 0 & 1 & 1 \\
Africa & 0 & 0 & 0 & 1 & 0 & 0 \\
South America & 1 & 0 & 1 & 0 & 0 & 0 \\
Central America & 1 & 0 & 0 & 0 & 1 & 0 \\
and Caribbean region & 1 & 1 & 0 & 0 & 0 & 0 \\
North America 1 & 0 & 0 & 0 & 0 & 1 & 1 \\
North America 2 & 1 & 1 & 1 & 1 & 1 & 1 \\
West Europe & 0 & 1 & 1 & 1 & 1 & 1 \\
East Europe & 1 & 1 & 0 & 0 & 1 & 1 \\
South Asia & 0 & 1 & 1 & 0 & 1 & 1 \\
West Asia & 1 & 0 & 0 & 1 & 1 & 1 \\
East Asia & 0 & 0 & 0 & 1 & 1 & 1 \\
North Asia & & & 0 & & 1 \\
\hline
\end{tabular}

Note: (1) presence and (0) absence of the species group in a region. 
$R C=0.27$. The consensus tree obtained by the MajRule method is shown in Fig. 1a.

The dendrogram indicates that the center of the genus origin was at the interface of North America, South America, Africa, Europe, and Asia. This implies that the genus originated from the center of the paleocontinent Pangea at the interface of the continental plates (Carbon, 350-285 Myr) (Fig. 1b). The part of the genus that remained on the Laurasian (northern) plate of Pangea was divided into the West-Asian and NorthAsian faunae. The European fauna originated from the West Asian branch, the East Asian and North Asian branches originated from the South Asian (South China) fauna. The East Asian branch of Bursaphelenchus, having formed specific combinations of various species groups, spread, during the Tertiary period, through the Bering Strait and populated North America from the north. The dendrogram-based model of the genus expansion can be seen in Fig. 1b. The distribution of both lower (hunti, aberrans) and higher (xylophilus, piniperdae) species groups confirms these pathways of historical migration. The most similar faunae (at the level of species groups) were at the interface of paleocontinents (outlined with a hatched ellipse in Fig. 1b).

The morphological species groups hunti and xylophilus were previously shown to be the most verifiable on the basis of the taxa of insect vectors; therefore, they can be accepted as natural species groups within the genus Bursaphelenchus [1]. Five out of six species of the hunti group are found in America (B. hunti, B. gonzalezi, B. kevini, B. seani, and B. cocophilus), and two of them (B. dongguanensis and $B$. hunti) have also been recorded in East Asia. This suggests an American origin of the group.

Six out of ten species of the xylophilus group are found in East Asia. The East Asian species (B. kolymensis, B. conicaudatus, B. mucronatus, and B. ershenkii) are located at the basis of the cluster 1 branch (xylophilus) in the genus dendrogram [1]. Four species are found in Europe (B. xylophilus has been introduced by humans), and four species in North America. The group appeared in the northern Laurasian shield of Pangea (Carbon) in the place of the modern East Asia. Part of the species populated America through the Euroamerican part of Pangea. The B. xylophilus species formation occurred in associations with the resident coniferous plants and longhorn beetles of the family Cerambycidae in North America. On the Asian continent, this species appeared due to anthropogenic spread (from the United States to Japan and China) [7, 8]. B. xylophilus populated coniferous forests of Asia because adaptations of this species are similar to those of the resident Bursaphelenchus species. In East Asia, other species of the xylophilus group are distributed, and suitable host plants and vectors (longhorn beetles) are available. American conifers have adapted to B. xylophilus, and nematode parasitism is not fatal for the trees. In South
Asia, this species has proved to be well-adapted to populating forests; however, the parasite-host system has appeared to be instable; therefore, Asian conifers die from B. xylophilus. Thus, this is a species of world plant quarantine importance. At the same time, $B$. mucronatus from the same group but of Eurasian origin caused significant damage to North American conifers, which was confirmed experimentally [9]. In Europe, a local focus of B. xylophilus is in Portugal, in a $100-\mathrm{km}$ quarantine zone around the port Setúbal on the Atlantic coast. Portugal researchers have speculated that B. xylophilus has been introduced from East Asia to Portugal via the sea route [10]. It could occur during evacuation of the Portugal colony Macao in 19971998.

Thus, the genus Bursaphelenchus may have formed in the center of Pangea, at the interface of the continental plates (Carbon); the hunti group is presumably of American origin, the xylophilus group appeared in the northern Laurasian shield of Pangea (during Carbon) in the area of the modern East Asia; B. xylophilus, a species of world quarantine importance, developed in North America and afterwards expanded to coniferous forests of East Asia due to similarity with the resident Bursaphelenchus species with respect to adaptations: in East Asia, other species of the xylophilus group are distributed, and suitable hosts and vectors (longhorn beetles) are available.

\section{REFERENCES}

1. Ryss, A., Vieira, P., Mota, M., and Kulinich, O., Nematology, 2005, vol. 7, no. 3, pp. 393-458.

2. OEPP/EPPO. EPPO A1 and A2 Lists of Pests Recommended for Regulation as Quarantine Pests, European and Mediterranean Plant Protection Organization, 2006 (http://www.eppo.org/quarantine/)

3. Brooks, D.R., Van Veller, M.G.P., and McLennan, D.A., J. Biogeogr., 2001, vol. 28, pp. 345-358.

4. Brooks, D.R. and McLennan, D.A., Cladistics, 2003, vol. 19, pp. 104-119.

5. Halas, D., Zamparo, D., and Brooks, D.R., J. Biogeogr., 2005, vol. 32, pp. 249-260.

6. Swofford, D.L., PAUP: Phylogenetic Analysis Using Parsimony. Vers. 4.0b10, Sunderland: Sinauer, 2001.

7. Robbins, K., in Proc. National Pine Wilt Disease Workshop III, Champaign: Nat. Hist. Surv., 1982, pp. 3-6.

8. Rutherford, T.A., Mamiya, Y., and Webster, J.M., Forest Sci., 1990, vol. 36, pp. 145-155.

9. Mamiya, Y., in Sustainability of Pine Forests in Relation to Pine Wilt and Decline (Proc. Symposium), Kyoto: Shokado Shoten, 1999, pp. 57-63.

10. Mota, M.M., Braasch, H., Bravo, M.A., et al., Nematology, 1999, vol. 1, pp. 727-734.

11. Ushakov, S.A. and Yasamanov, N.A., Dreif materikov $i$ klimaty Zemli (Continental Drift and Earth's Climates), Moscow: Mysl', 1984. 